\title{
An integrative review of assessments used in occupational therapy interventions for children with cerebral palsy
}

\author{
Carly Peters $^{a}$ (D) , Amy Chang ${ }^{a}$ (D), Abbiagail Morales ${ }^{a}$ (D), Karin Barnes ${ }^{b}$ (D), \\ Ana Allegretti ${ }^{\mathrm{b}}$ (1)
}

aProgram at the UT Health San Antonio, Texas, United States.

boccupational Therapy Department, UT Health San Antonio, Texas, United States.

\begin{abstract}
Introduction: Children with cerebral palsy (CP) experience a wide range of deficits and symptoms. When undergoing occupational therapy (OT) interventions, it is essential that the OT select assessments that can accurately reflect the outcome measures of the targeted domains. Objective: To identify the assessment tools most frequently reported in research studies as measures for OT interventions when treating children with CP. Method: Pubmed and Ovid databases were systematically searched by using key terms "cerebral palsy," "assessments," and "occupational therapy". Assessment tools were explored and extracted from articles contingent on the following inclusion criteria: (1) children birth to 18 years diagnosed with cerebral palsy; (2) use of assessment(s) as a measure of OT intervention; (3) published in English between 2007 and 2017. In the preliminary search, Pubmed yielded 151 records and Ovid yielded 571. Out of these, only 76 met the inclusion criteria. From the remaining 76 articles, a total of 88 assessment tools were retrieved and included in this literature review. Results: Ten assessments were found to be of importance based on frequency of use. The Assisting Hand Assessment (AHA) and Pediatric Evaluation of Disability Inventory (PEDI) were the most commonly used. Conclusion: This study helps to determine which assessments are frequently used in OT practice with children with CP. The findings of this study play an important role in addressing the challenge of assessment selection faced by occupational therapists and provide a basis for future research to expand on with regards to treating children with CP.
\end{abstract}

Keywords: Cerebral Palsy, Occupational Therapists, Assessments.

\section{Revisáo integrativa de avaliaçóes utilizadas em intervençóes de terapia ocupacional para crianças com paralisia cerebral}

Resumo: Introdução: Crianças com Paralisia Cerebral (PC) apresentam uma ampla gama de déficits e sintomas. Quando submetidos a intervenções de terapia ocupacional (OT), é essencial que as avaliações utilizadas reflitam com precisão as medidas de resultados dos domínios visados. Objetivo: Identificar os instrumentos de avaliação mais utilizados como medidas para intervenções no TO no tratamento de crianças com PC. Método: Bancos de dados Pubmed e Ovid foram sistematicamente pesquisados usando os termos-chave "paralisia cerebral", "avaliações" e "terapia ocupacional”. Avaliaçoes foram exploradas e extraídas de artigos contingentes aos seguintes critérios de inclusão: (1) crianças de até 18 anos com diagnóstico de paralisia cerebral; (2) uso da(s) avaliação (ões) como medida de intervenção do TO; (3) publicado em inglês entre 2007 e 2017. Na pesquisa preliminar, foram encontrados 151 artigos em Pubmed e 571 em Ovid. Destes, apenas 76 foram inlcuidos devido aos critérios de inclusão. Dos 76 artigos restantes, um total de 89 avaliaçoes foram encontradas e incluídas nesta revisão da literatura. Resultados: Dez avaliações foram consideradas importantes com base na frequência de uso. A “Assisting Hand Assessment” (AHA) e o Inventário de Avaliação Pediátrica de Incapacidade (PEDI) foram os mais utilizados.

Corresponding author: Ana Allegretti, Department at UT Health San Antonio, Texas, United States, e-mail: allegrettial@uthscsa.edu Received on Nov. 20, 2018; $1^{\text {st }}$ Revision on Dec 20, 2018; Accepted on Jan. 10, 2019.

This is an Open Access article distributed under the terms of the Creative Commons Attribution License, which permits unrestricted use, distribution, and reproduction in any medium, provided the original work is properly cited. 
Conclusão: Os achados deste estudo desempenham um papel importante no e do desafio de seleção de avaliação enfrentado pelos terapeutas ocupacionais e fornecem uma base para futuras pesquisas para expandir no que diz respeito ao tratamento de crianças com PC.

Palavras-chave: Paralisia Cerebral, Terapeutas Ocupacionais, Avaliações.

\section{Introduction}

Cerebral palsy $(\mathrm{CP})$ is a non-progressive group of developmental motor disorders that may result in spasticity, paralysis, or abnormal posture (COKER-BOLT; GARCIA; NABER, 2015). $\mathrm{CP}$ affects motor skills, movement, and muscle tone and is the most common cause of physical disability in childhood with a consistent prevalence of 3.1 to 3.6 per 1000 births for over the last 20 years (LOWES et al., 2014; CHRISTENSEN et al., 2014). Because CP is a lifelong condition, all aspects of an individual's development and independence are impacted (STEULTJENS et al., 2004). Occupational therapy (OT) is one of many disciplines that provides treatment for children with $\mathrm{CP}$ with the goal of optimizing functional abilities and increasing independence (STEULTJENS et al., 2004). OT focuses on skill development to perform activities of daily living, addresses cognitive and perceptual deficits, promotes functional independence, and utilizes a wide variety of interventions and approaches during treatment (STEULTJENS et al., 2004). The American Occupational Therapy Association's centennial vision has caused a rising demand for practitioners to provide evidence-based treatment in order to maximize effectiveness of client care (AMERICAN..., 2017; SALEH et al., 2009). A major challenge in basing OT interventions on evidence-based practice is choosing the most appropriate assessment for individual clients (SALEH et al., 2009). Specifically, OTs report that standardized assessment, which are essential to OT practice, benefit clients and the OT profession as opposed to non-standardized assessments (PIERNIK-YODER; BECK, 2012). Considering the specific and complex deficits and needs of children with CP, it is essential that standardized assessments utilized to measure the unique skills and behaviors of this population accurately align with and reflect targeted outcomes when undergoing OT interventions. According to Wright and Majnemer (2014), if all clinicians in the rehabilitative community chose assessments from the same toolbox when treating children with CP, then a universal approach of measuring outcomes can be developed. While Wright and Majnemer (2014) discussed concepts that should be considered when choosing assessments to use with children with CP, they only provided examples of possible measures clinicians can utilize.

Other research that has been conducted to review the literature on assessment tools used for children with CP include Wagner and Davids (2012) systematic review study. Using the databases Health and Psychosocial Instruments (HaPI), US National Library of Medicine (PubMed), and Cumulative Index to Nursing and Allied Health Literature (CINAHL Plus) databases, they found twenty-one assessment tools and classification systems used specifically for upper extremity function and performance in children with CP (WAGNER; DAVIDS, 2012). Furthermore, a systematic review that searched MEDLINE, Embase, CINAHL, and PscyhINFO databases found eight assessments that were used to measure only activity limitation for children with CP (HARVEY et al., 2008). Functional motor abilities of children with CP represented another domain used to conduct a systematic literature review of assessment measures (KETELAAR; VERMEER; HELDERS, 1998). The study searched MEDLINE, Sportdisk, and PsychLIT databases and yielded seventeen instruments used in pediatric rehabilitation to evaluate functional motor abilities of children with CP (KETELAAR; VERMEER; HELDERS, 1998).

Overall, these studies showed the types of assessments being used to measure specific domains (DIAS et al., 2017). However, there remains a lack of evidence on what assessments are currently being used to measure outcomes specifically for OT interventions for children with CP. In order to develop a toolbox of assessments utilized for children with $\mathrm{CP}$, it is necessary to gain a cohesive understanding of the current assessments in use. The purpose of this study was to perform an integrative review of the literature to identify what assessments are being used to measure outcomes of OT interventions for children with CP.

\section{Method}

The study aimed to obtain a frequency count of each assessment used with the children with CP as reported the literature. An integrative review was performed and followed Arksey and O'Malley (2005) stages of methodological framework, which 
include identifying the research question, identifying relevant studies, selecting studies, charting the data, and summarizing and reporting the results (Figure 1). The research team included three student investigators and a supervising professor. Pubmed and Ovid databases were systematically searched in January 2018 using the key terms "cerebral palsy", "assessment" and "occupational therapy". The following inclusion criteria were used: (1) children birth to 18 years old diagnosed with cerebral palsy; (2) use of assessment(s); (3) published in English between 2007 and 2017. Using these criteria, our search yielded a total of 383 articles that were imported to RefWorks. After eliminating duplicate articles, 307 articles remained and were allocated between three student researchers to be further reviewed by abstract and title. Based off the content found, further exclusion criteria were developed post hoc and included (1) articles lacking OT intervention; (2) systematic reviews. Assessments used in systematic reviews were not included in the frequency count to eliminate the possibility of duplicates. With the use of these post hoc criteria 76 articles were included for the analysis in this integrative review.

\section{Results}

The search yielded 76 articles that were synthesized and analyzed. In table 1, the list of the 76 articles and the assessments reported in each article are presented. The researchers found that there are 88 different assessments presented in these studies,

Of the 88 assessments, the ten most frequently reported in the studies are in descending order, including the Assisting Hand Assessment (AHA), Pediatric Evaluation of Disability Inventory (PEDI), Gross Motor Function Classification Scale (GMFCS), Canadian Occupational Performance Measure (COPM), Goal Attainment Scale (GAS), Manual Ability Classification System (MACS),
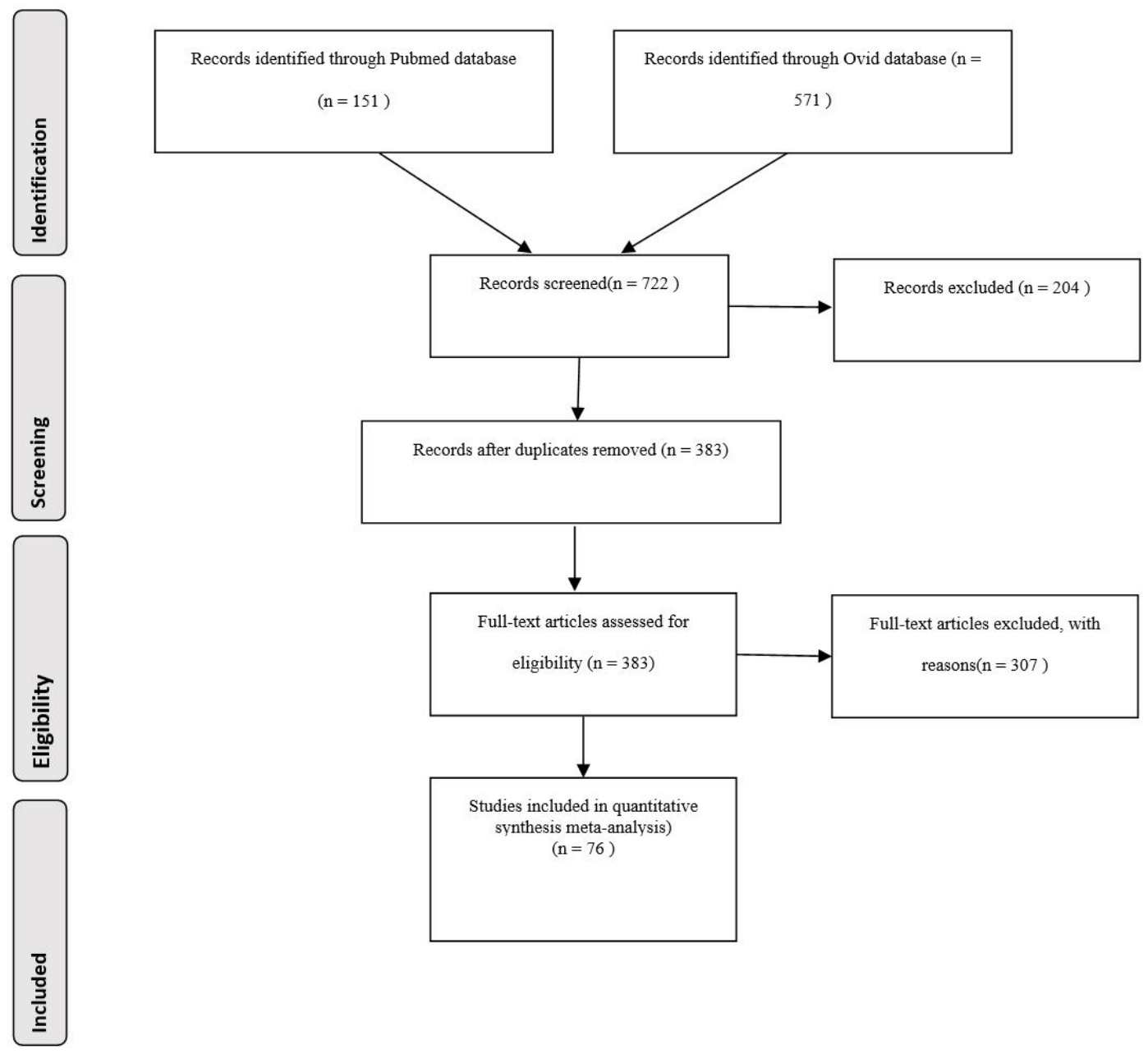

Figure 1. Flow chart of search process. 
Table 1. Assessments Used for Children with CP as Found in Literature.

\begin{tabular}{llcl}
\hline & Author, Year & Assessments \\
\hline Aarts et al. (2007) & & VOAA &
\end{tabular}

https://doi.org/10.1002/oti.229

Aarts et al. (2012)

https://doi.org/10.1002/oti.321

Auld et al. (2012)

https://doi.org/10.3109/01942638.2011.572150

Bailes, Greve and Schmitt (2010)

https://doi.org/10.1097/PEP.0b013e3181cbf224

Bailes et al. (2011)

https://doi.org/10.1097/PEP.0b013e318218ef58

Barroso et al. (2011)

https://doi.org/10.1016/j.clinbiomech.2011.05.006

Berge et al. (2012)

https://doi.org/10.1177/0269215511411936

Bleyenheuft et al. (2015)

https://doi.org/10.1177/1545968314562109

Bleyenheuft et al. (2017)

https://doi.org/10.1111/dmcn.13338

Brandão, Gordon and Mancini (2012)

https://doi.org/10.5014/ajot.2012.004622

Brandao et al. (2010)

https://doi.org/10.1177\%2F0269215510367974

Buccino et al. (2012)

https://doi.org/10.1111/j.1469-8749.2012.04334.x

Cameron et al. (2017)

https://doi.org/10.1080/01942638.2016.1185500

Case-Smith et al. (2012)

10.5014/ajot.2012.002386

Chen et al. (2014)

https://doi.org/10.5014/ajot.2014.009860

Cohen-Holzer et al. (2016)

https://doi.org/10.3109/01942638.2014.990549

Coker-Bolt et al. (2015)

10.5014/ajot.2016.70S1-PO5115
AHA, ABILITHAND-Kids, COPM, GAS, VOAA, Melbourne

Disk-Criminator, Exteroception, Klingel's method, SIPT, SWMs, NSMDA

GMFM-66, PEDI

GMFM-66, PEDI

JTTHF, Digital image acquisition system for range of motion

GAS, House classification, MACS, VAS

ABILIHAND-Kids, ABILICO-kids, AHA, BBT, PEDI, LIFE-H, 6MWT, Pinch

ABILIHAND-Kids

COPM, PEDI

JTTHF, PEDI

Melbourne

COPM, PQRS

AHA, QUEST, PMAL

PDMS-2, PMAL, WeeFIM

AHA, JTTHF

CHUEQ, MA2, PEDI

Note: $[6 \mathrm{MWT}=$ Six-Minute Walk Test; AHA = Assisting Hand Assessment; AIMS = Alberta Infant Motor Scale; AMPS $=$ Assessment of Motor and Process Skills; APCP $=$ Assessment of Preschool Children's Participation; BBT $=$ Box and Block Test; BFMF = Bimanual Fine Motor Function scale; BOT = Bruininks-Oseretsky Test of Motor Proficiency; $\mathrm{CAPE}=$ Children's Assessment of Participation and Enjoyment; $\mathrm{CBC}=$ Child Behavior Checklist CFUS $=$ Caregiver Functional Use Survey; CHEQ; Children's Hand-Use Experience Questionnaire; CHQ = Australian Authorised Adaptation of the Child Health Questionnaire; CHUEQ = Children's Hand-Use Experience Questionaire; COPM = Canadian Occupational Performance Meausure; CPQOL-Child = Cerebral Palsy Quality of Life Questionnaire for Children; ENNAS = Einstein Neonatal Neurobehavioral Assessment Scale; ETCH = Evaluation Tool of Children's Handwriting; FES Family Empowerment Scale; fTORT = functional Tactile Object Recognition Test; GAS = Goal Attainment Scale; GMA = General Movements Assessment; GMFCS $=$ Gross Motor Function Classification System; GMFM = Gross Motor Function Measure; GMFM- $66=$ Gross Motor Function Measure - 66; GMFM-88 = Gross Motor Function Measure - 88; ICFI = International Classification of Functioning Interview; JTTHF = Jebsen-Taylor Hand Function Test; LIFE-H = Assessment of Life Habits; MA2 = Melbourne Assessment 2; MACS = Manual Ability Classification System; MAS = Modified Ashworth Scale; Melbourne $=$ Melbourne Assessment of Unilateral Upper Limb Function; MHA = Minnesota Handwriting Assessment; NHDC = Neurological Hand Deformity Classification; NSMDA $=$ Neurosensory Motor Developmental Assessment; PAC $=$ Preferences for Activity of Children; PAFT $=$ Pediatric Arm Function Test; PDMS-2 = Peabody Developmental Motor Scales II; PEDI = Pediatric Evaluation of Disability Inventory; PedsQL = Pediatric Quality of Life Inventory; PGMS = Peabody Gross Motor Scale; PMAL = Pediatric Motor Activity Log; PQRS = Performance Quality Rating Scale; PRT = Pediatric Reach Test; PSI-Short form = Parenting Stress Index-Short Form; QUEST = Quality of Upper Extremity Skills Test; ROM = Range of Motion; SAS = Sitting Assessment Scale; SIPT = Sensory Integration and Praxis Test; SPPC = Harter Self-Perception Profile; SSC = Sense and Self-Regulation Checklist; SWMs = Semmes Weinstein Monofilaments; TAUT = Toddler Arm Use Test; TCMS = Trunk Control Measurement Scale; THS-R = Test of Handwriting Skills-Revised; TIMP = Test of Infant Motor Performance; TIS = Trunk Impairment Scale; TVPS3 = Test of Visual Perceptual Skill -non-motor; VAS = Visual Analogue Scale; VMI = Beery-Buktenica Developmental Test of Visual-Motor Integration; VOAA = Video Observations Aarts and Aarts]. 
Table 1. Continued...

\begin{tabular}{|c|c|}
\hline Author, Year & Assessments \\
\hline $\begin{array}{l}\text { DeLuca et al. (2012) } \\
\text { https://doi.org/10.3233/PRM-2012-0206 }\end{array}$ & AHA, QUEST, PMAL Shriners Hospital \\
\hline $\begin{array}{l}\text { Ferre et al. (2017) } \\
\text { https://doi.org/10.1111/dmcn. } 13330\end{array}$ & AHA, BBT, COPM \\
\hline $\begin{array}{l}\text { Gelkop et al. (2015) } \\
\text { https://doi.org/10.3109/01942638.2014.925027 }\end{array}$ & AHA, QUEST \\
\hline $\begin{array}{l}\text { Georgiades et al. (2014) } \\
\text { https://doi.org/10.1111/1440-1630.12150 }\end{array}$ & NHDC \\
\hline $\begin{array}{l}\text { Golomb et al. (2010) } \\
\text { https://doi.org/10.1016/j.apmr.2009.08.153 }\end{array}$ & BOT, JTTHF, Dynamometer, Pinch \\
\hline $\begin{array}{l}\text { Gordon et al. (2007) } \\
\text { https://doi.org/10.1111/j.1469-8749.2007.00830.x }\end{array}$ & AHA, Accelerometry, BOT, CFUS, JTTHF \\
\hline $\begin{array}{l}\text { Hamil, Washington and White (2007) } \\
\text { https://doi.org/10.1080/J006v27n04_03 }\end{array}$ & GMFCS, GMFM, SAS \\
\hline $\begin{array}{l}\text { Hansen et al. (2012) } \\
\text { https://doi.org/10.1177\%2F2156587211430833 }\end{array}$ & CBC, GMFM-66, ICFI \\
\hline $\begin{array}{l}\text { Hoare et al. (2013) } \\
\text { https://doi.org/10.1111/dmcn. } 12054\end{array}$ & AHA, COPM, GAS, QUEST, PEDI \\
\hline $\begin{array}{l}\text { Hoare et al. (2010) } \\
\text { https://doi.org/10.1186/1471-2377-10-58 }\end{array}$ & $\begin{array}{l}\text { AHA, COPM, GAS, QUEST, PEDI, MAS, } \\
\text { Modified Tardieu Scale, PMAL }\end{array}$ \\
\hline $\begin{array}{l}\text { Houwink et al. (2013) } \\
\text { https://doi.org/10.1111/j.1469-8749.2012.04442.x }\end{array}$ & VOAA \\
\hline $\begin{array}{l}\text { Howcroft et al. (2011) } \\
\text { https://doi.org/10.1111/j.1469-8749.2011.04078.x }\end{array}$ & AHA \\
\hline $\begin{array}{l}\text { Huang et al. (2014) } \\
\text { https://doi.org/10.1097/PEP.0000000000000001 }\end{array}$ & PEDI \\
\hline $\begin{array}{l}\text { Imms et al. (2017) } \\
\text { https://doi.org/10.1111/dmcn.13302 }\end{array}$ & CAPE, PAC \\
\hline $\begin{array}{l}\text { James et al. }(2015 \mathrm{a}) \\
\mathrm{https}: / / \text { doi.org/10.1111/dmcn.12705 }\end{array}$ & $\begin{array}{l}\text { AHA, AMPS, COPM, JTTHF, Melbourne, TVPS- } \\
3\end{array}$ \\
\hline James et al. (2015b) & AMPS \\
\hline
\end{tabular}
https://doi.org/10.3109/01942638.2015.1076555

Kara et al. (2015)

https://doi.org/10.1111/dmen.12583

BOT, BFMF, GMFM, GMFCS, MACS, WeeFIM

Note: $[6 \mathrm{MWT}=$ Six-Minute Walk Test; AHA = Assisting Hand Assessment; AIMS = Alberta Infant Motor Scale; AMPS = Assessment of Motor and Process Skills; APCP = Assessment of Preschool Children's Participation; BBT = Box and Block Test; BFMF = Bimanual Fine Motor Function scale; BOT = Bruininks-Oseretsky Test of Motor Proficiency; $\mathrm{CAPE}=$ Children's Assessment of Participation and Enjoyment $\mathrm{CBC}=$ Child Behavior Checklist CFUS $=$ Caregiver Functional Use Survey; CHEQ; Children's Hand-Use Experience Questionnaire; CHQ = Australian Authorised Adaptation of the Child Health Questionnaire; CHUEQ = Children's Hand-Use Experience Questionaire; COPM = Canadian Occupational Performance Meausure; CPQOL-Child = Cerebral Palsy Quality of Life Questionnaire for Children; ENNAS = Einstein Neonatal Neurobehavioral Assessment Scale; ETCH = Evaluation Tool of Children's Handwriting; FES Family Empowerment Scale; fTORT = functional Tactile Object Recognition Test; GAS = Goal Attainment Scale; GMA = General Movements Assessment; GMFCS $=$ Gross Motor Function Classification System; GMFM = Gross Motor Function Measure; GMFM- $66=$ Gross Motor Function Measure - 66; GMFM-88 = Gross Motor Function Measure - 88; ICFI = International Classification of Functioning Interview; JTTHF = Jebsen-Taylor Hand Function Test; LIFE-H = Assessment of Life Habits; MA2 = Melbourne Assessment 2; MACS = Manual Ability Classification System; MAS = Modified Ashworth Scale; Melbourne = Melbourne Assessment of Unilateral Upper Limb Function; MHA = Minnesota Handwriting Assessment; NHDC = Neurological Hand Deformity Classification; NSMDA = Neurosensory Motor Developmental Assessment; PAC $=$ Preferences for Activity of Children; PAFT = Pediatric Arm Function Test; PDMS-2 = Peabody Developmental Motor Scales II; PEDI = Pediatric Evaluation of Disability Inventory; PedsQL = Pediatric Quality of Life Inventory; PGMS = Peabody Gross Motor Scale; PMAL = Pediatric Motor Activity Log; PQRS = Performance Quality Rating Scale; PRT = Pediatric Reach Test; PSI-Short form = Parenting Stress Index-Short Form; QUEST = Quality of Upper Extremity Skills Test; ROM = Range of Motion; SAS = Sitting Assessment Scale; SIPT = Sensory Integration and Praxis Test; SPPC = Harter Self-Perception Profile; SSC = Sense and Self-Regulation Checklist; SWMs = Semmes Weinstein Monofilaments; TAUT = Toddler Arm Use Test; TCMS = Trunk Control Measurement Scale; THS-R = Test of Handwriting Skills-Revised; TIMP = Test of Infant Motor Performance; TIS = Trunk Impairment Scale; TVPS3 = Test of Visual Perceptual Skill -non-motor; VAS = Visual Analogue Scale; VMI = Beery-Buktenica Developmental Test of Visual-Motor Integration; VOAA = Video Observations Aarts and Aarts]. 
Table 1. Continued...

\begin{tabular}{ll}
\hline \multicolumn{1}{c}{ Author, Year } & \multicolumn{1}{c}{ Assessments } \\
\hline $\begin{array}{l}\text { Kirkpatrick et al. (2016) } \\
\text { https://doi.org/10.1111/dmcn.13109 }\end{array}$ & ABILIHANDS-kids, AHA, MA2 \\
$\begin{array}{l}\text { Kruijsen-Terpstra et al. (2016) } \\
\text { https://doi.org/10.1111/dmcn.12966 }\end{array}$ & APCP, COPM, GAS, GMFCS, GMFM-66, \\
& MACS, PEDI-CAS, PEDI-FSS, Nijmeegse \\
Law et al. (2011) & Ouderlijke Stress Index- Kort \\
https://doi.org/10.1111/j.1469-8749.2011.03962.x & APCP, GMFCS, GMFM-66, PEDI, ROM, Family \\
Lidman et al. (2015) & Empowerment Scale \\
https://doi.org/10.1111/dmcn.12739 & AHA, COPM, ROM \\
Lin et al. (2011) & \\
\end{tabular}

https://doi.org/10.1016/j.ridd.2011.01.023

Louwers et al. (2011)

https://doi.org/10.1111/j.1469-8749.2010.03849.x

Luna-Oliva et al. (2013)

https://doi.org/10.3233/NRE-131001

Mackey et al. (2008)

https://doi.org/10.1111/j.1468-1331.2008.02271.x

Mclean et al. (2017)

https://doi.org/10.5014/ajot.2016.024968

Man and Wong (2007)

https://doi.org/10.5014/ajot.61.3.355

Matusiak-Wieczorek et al. (2016)

AHA, GMFCS, MACS, Zancolli classification, House classification

AMPS, GMFCS, GMFM, JTTHF, PRT

MAS, Melbourne, 3-D Kinematics

https://doi.org/10.5604/15093492.1205024

McConnell, Johnston and Kerr (2014)

https://doi.org/10.3109/01942638.2013.866611

McGarry, Moir and Girdler (2012)

https://doi.org/10.3109/17483107.2011.637283

Millard, Benore and Mosher (2013)

https://doi.org/10.1037/cpp0000005

Novak, Cusick and Lowe (2007)

http://doi.org/10.5014/ajot.61.4.463

Ostensjø, Oien and Fallang (2008)

https://doi.org/10.1080/17518420802525500

Palsbo and Hood-Szivek (2012)

https://doi.org/10.5014/ajot.2012.004556

AHA, BBT, COPM, GAS, fTORT, Sense-Assess

kids, Wrist position test

Assessment of Comfort, WinFitts

GMFCS, SAS

Melbourne

Powered Mobility Program Assessment Battery, GMFCS

GMFCS, WeeFIM

GAS, PEDI, QUEST

COPM, GAS

ETCH, THS-R, Print tool, VMI

Note: $[6 \mathrm{MWT}=$ Six-Minute Walk Test; AHA = Assisting Hand Assessment; AIMS = Alberta Infant Motor Scale; AMPS = Assessment of Motor and Process Skills; APCP = Assessment of Preschool Children's Participation; BBT = Box and Block Test; BFMF = Bimanual Fine Motor Function scale; BOT = Bruininks-Oseretsky Test of Motor Proficiency; $\mathrm{CAPE}=$ Children's Assessment of Participation and Enjoyment $\mathrm{CBC}=$ Child Behavior Checklist; CFUS $=$ Caregiver Functional Use Survey; CHEQ; Children's Hand-Use Experience Questionnaire; CHQ = Australian Authorised Adaptation of the Child Health Questionnaire; CHUEQ = Children's Hand-Use Experience Questionaire; COPM = Canadian Occupational Performance Meausure; CPQOL-Child = Cerebral Palsy Quality of Life Questionnaire for Children; ENNAS = Einstein Neonatal Neurobehavioral Assessment Scale; ETCH = Evaluation Tool of Children's Handwriting; FES Family Empowerment Scale; fTORT = functional Tactile Object Recognition Test; GAS = Goal Attainment Scale; GMA = General Movements Assessment; GMFCS = Gross Motor Function Classification System; GMFM = Gross Motor Function Measure; GMFM- 66 = Gross Motor Function Measure - 66; GMFM-88 = Gross Motor Function Measure - 88; ICFI = International Classification of Functioning Interview; JTTHF = Jebsen-Taylor Hand Function Test; LIFE-H = Assessment of Life Habits; MA2 = Melbourne Assessment 2; MACS = Manual Ability Classification System; MAS = Modified Ashworth Scale; Melbourne = Melbourne Assessment of Unilateral Upper Limb Function; MHA = Minnesota Handwriting Assessment; NHDC = Neurological Hand Deformity Classification; NSMDA = Neurosensory Motor Developmental Assessment; PAC $=$ Preferences for Activity of Children; PAFT = Pediatric Arm Function Test; PDMS-2 = Peabody Developmental Motor Scales II; PEDI = Pediatric Evaluation of Disability Inventory; PedsQL = Pediatric Quality of Life Inventory; PGMS = Peabody Gross Motor Scale; PMAL = Pediatric Motor Activity Log; PQRS = Performance Quality Rating Scale; PRT = Pediatric Reach Test; PSI-Short form = Parenting Stress Index-Short Form; QUEST = Quality of Upper Extremity Skills Test; ROM = Range of Motion; SAS = Sitting Assessment Scale; SIPT = Sensory Integration and Praxis Test; SPPC = Harter Self-Perception Profile; SSC = Sense and Self-Regulation Checklist; SWMs = Semmes Weinstein Monofilaments; TAUT = Toddler Arm Use Test; TCMS = Trunk Control Measurement Scale; THS-R = Test of Handwriting Skills-Revised; TIMP = Test of Infant Motor Performance; TIS = Trunk Impairment Scale; TVPS3 = Test of Visual Perceptual Skill -non-motor; VAS = Visual Analogue Scale; VMI = Beery-Buktenica Developmental Test of Visual-Motor Integration; VOAA = Video Observations Aarts and Aarts]. 
Table 1. Continued...

\begin{tabular}{|c|c|}
\hline Author, Year & Assessments \\
\hline $\begin{array}{l}\text { Pham et al. (2016) } \\
\text { https://doi.org/10.3109/01942638.2015.1127867 }\end{array}$ & GMFCS, GMFM-66, TCMS, TIS \\
\hline $\begin{array}{l}\text { Phipps and Roberts (2012) } \\
\text { https://doi.org/10.5014/ajot.2012.003921 }\end{array}$ & GMFCS, MACS, PEDI \\
\hline $\begin{array}{l}\text { Psychouli and Kennedy (2016) } \\
\text { https://doi.org/10.1097/PEP.0000000000000227 }\end{array}$ & Melbourne, QUEST \\
\hline $\begin{array}{l}\text { Robert et al. (2013) } \\
\text { https://doi.org/10.1111/dmcn.12219 }\end{array}$ & MACS, Melbourne, SWMs, UE PROM \\
\hline $\begin{array}{l}\text { Rostami et al. (2012) } \\
\text { https://doi.org/10.3233/NRE-2012-00804 }\end{array}$ & BOT, MAS, PMAL \\
\hline $\begin{array}{l}\text { Ryan, Rigby and Campbell (2010) } \\
\text { https://doi.org/10.1111/j.1440-1630.2009.00831.x }\end{array}$ & GMFCS, MHA \\
\hline $\begin{array}{l}\text { Ryll, Bastiaenen and Eliasson (2017) } \\
\text { https://doi.org/10.1080/01942638.2016.1185498 }\end{array}$ & AHA, CHEQ, MACS \\
\hline $\begin{array}{l}\text { Sakzewski et al. (2011) } \\
\text { https://doi.org/10.1177/1545968311400093 }\end{array}$ & AHA, COPM, JTTHF, LIFE-H, MAS, Melbourne \\
\hline $\begin{array}{l}\text { Sakzewski et al. (2012) } \\
\text { https://doi.org/10.1111/j.1469-8749.2012.04272.x }\end{array}$ & CPQOL, MACS \\
\hline $\begin{array}{l}\text { Sakzewski et al. (2015) } \\
\text { https://doi.org/10.1111/dmcn.12702 }\end{array}$ & AHA, MACS \\
\hline $\begin{array}{l}\text { Schneiberg et al. (2010) } \\
\text { https://doi.org/10.1111/j.1469-8749.2010.03768.x }\end{array}$ & $\begin{array}{l}\text { CSI, Disk-Criminator, Light touch/position, } \\
\text { Melbourne, SWMs }\end{array}$ \\
\hline $\begin{array}{l}\text { Schrank (2013) } \\
\text { https://doi.org/10.1097/PEP.0b013e31827abaf4 }\end{array}$ & GMFM-88, WeeFIM \\
\hline $\begin{array}{l}\text { Silva et al. (2012) } \\
\text { https://doi.org/10.5014/ajot.2012.003541 }\end{array}$ & PGMS, SSC \\
\hline $\begin{array}{l}\text { Snider et al. (2008) } \\
\text { https://doi.org/10.1016/j.earlhumdev.2007.07.004 }\end{array}$ & AIMS, ENNAS, GMA, TIMP \\
\hline $\begin{array}{l}\text { Steenbeek et al. }(2010) \\
\text { https://doi.org/10.1177/0269215511407220 }\end{array}$ & GAS, GMFCS, GMFM-66, MACS, PEDI \\
\hline $\begin{array}{l}\text { Storvold and Jahnsen (2010) } \\
\text { https://doi.org/10.1097/PEP.0b013e3181dbe379 }\end{array}$ & $\begin{array}{l}\text { AHA, Dynamometer, GAS, GMFCS, GMFM-66, } \\
\text { PEDI }\end{array}$ \\
\hline $\begin{array}{l}\text { Thompson et al. (2015) } \\
\text { https://doi.org/10.1097/PEP.0000000000000111 }\end{array}$ & Dynamometer, QUEST, PEDI, ROM \\
\hline
\end{tabular}

Note: [6MWT = Six-Minute Walk Test; AHA = Assisting Hand Assessment; AIMS = Alberta Infant Motor Scale; AMPS = Assessment of Motor and Process Skills; APCP = Assessment of Preschool Children's Participation; BBT = Box and Block Test; BFMF = Bimanual Fine Motor Function scale; BOT = Bruininks-Oseretsky Test of Motor Proficiency; $\mathrm{CAPE}=$ Children's Assessment of Participation and Enjoyment; $\mathrm{CBC}=$ Child Behavior Checklist CFUS = Caregiver Functional Use Survey; CHEQ; Children's Hand-Use Experience Questionnaire; CHQ = Australian Authorised Adaptation of the Child Health Questionnaire; CHUEQ = Children's Hand-Use Experience Questionaire; COPM = Canadian Occupational Performance Meausure; CPQOL-Child = Cerebral Palsy Quality of Life Questionnaire for Children; ENNAS = Einstein Neonatal Neurobehavioral Assessment Scale; ETCH = Evaluation Tool of Children's Handwriting; FES Family Empowerment Scale; fTORT = functional Tactile Object Recognition Test; GAS = Goal Attainment Scale; GMA = General Movements Assessment; GMFCS $=$ Gross Motor Function Classification System; GMFM = Gross Motor Function Measure; GMFM- $66=$ Gross Motor Function Measure - 66; GMFM-88 = Gross Motor Function Measure - 88; ICFI = International Classification of Functioning Interview; JTTHF = Jebsen-Taylor Hand Function Test; LIFE-H = Assessment of Life Habits; MA2 = Melbourne Assessment 2; MACS = Manual Ability Classification System; MAS = Modified Ashworth Scale; Melbourne = Melbourne Assessment of Unilateral Upper Limb Function; MHA = Minnesota Handwriting Assessment; NHDC $=$ Neurological Hand Deformity Classification; NSMDA $=$ Neurosensory Motor Developmental Assessment; PAC $=$ Preferences for Activity of Children; PAFT = Pediatric Arm Function Test; PDMS-2 = Peabody Developmental Motor Scales II; PEDI = Pediatric Evaluation of Disability Inventory; PedsQL = Pediatric Quality of Life Inventory; PGMS = Peabody Gross Motor Scale; PMAL = Pediatric Motor Activity Log; PQRS = Performance Quality Rating Scale; PRT = Pediatric Reach Test; PSI-Short form = Parenting Stress Index-Short Form; QUEST = Quality of Upper Extremity Skills Test; ROM = Range of Motion; SAS = Sitting Assessment Scale; SIPT = Sensory Integration and Praxis Test; SPPC = Harter Self-Perception Profile; SSC = Sense and Self-Regulation Checklist; SWMs = Semmes Weinstein Monofilaments; TAUT = Toddler Arm Use Test; TCMS = Trunk Control Measurement Scale; THS-R = Test of Handwriting Skills-Revised; TIMP = Test of Infant Motor Performance; TIS = Trunk Impairment Scale; TVPS3 = Test of Visual Perceptual Skill -non-motor; VAS = Visual Analogue Scale; VMI = Beery-Buktenica Developmental Test of Visual-Motor Integration; VOAA = Video Observations Aarts and Aarts]. 
Table 1. Continued...

\begin{tabular}{ll}
\hline \multicolumn{1}{c}{ Author, Year } & \multicolumn{1}{c}{ Assessments } \\
\hline $\begin{array}{l}\text { Uswatte et al. (2012a) } \\
\text { https://doi.org/10.1097/PHM.0b013e318269ec76 } \\
\text { Uswatte et al. (2012b) }\end{array}$ & MACS, PAFT, TAUT \\
https://doi.org/10.1037/a0028516 & MACS, PAFT, PMAL-R \\
Wallen et al. (2011) & \\
https://doi.org/10.1080/17518420701640897 & AHA, COPM, GAS, MAS, PMAL, Tardieu Scale \\
Wallen, O'Flaherty and Waugh (2007) & \\
https://doi.org/10.1016/j.apmr.2006.10.017 & COPM, CHQ, GAS, Melbourne, PEDI, QUEST, \\
Wallen et al. (2008) & Tardieu Scale \\
https://doi.org/10.1111/j.1469-8749.2011.04086.x & AHA, COPM, GAS, GMFCS, MACS, MAS, \\
Wang et al. (2017) & Melbourne, PMAL-R, Tardieu Scale \\
hattps:/doi.org/10.1016/j. & BBT, BOT-2, MA2, PMAL-R
\end{tabular}

https://doi.org/10.1016/j.apmr.2017.01.024

Yabunaka et al. (2011)

https://doi.org/10.1097/PHM.0b013e3181fc7ddf

Yasukawa and Uronis (2014)

http://doi.org/10.1097/JPO.0000000000000022

Ziebell et al. (2009)

https://doi.org/10.1111/j.1440-1630.2008.00775.x

\author{
GMFCS, GMFM-66
}

Melbourne

BOT, GMFCS, SPPC

\begin{abstract}
Note: $[6 \mathrm{MWT}=$ Six-Minute Walk Test; AHA = Assisting Hand Assessment; AIMS = Alberta Infant Motor Scale; AMPS = Assessment of Motor and Process Skills; APCP = Assessment of Preschool Children's Participation; BBT = Box and Block Test; BFMF = Bimanual Fine Motor Function scale; BOT = Bruininks-Oseretsky Test of Motor Proficiency; $\mathrm{CAPE}=$ Children's Assessment of Participation and Enjoyment; $\mathrm{CBC}=$ Child Behavior Checklist; CFUS $=$ Caregiver Functional Use Survey; CHEQ; Children's Hand-Use Experience Questionnaire; CHQ = Australian Authorised Adaptation of the Child Health Questionnaire; CHUEQ = Children's Hand-Use Experience Questionaire; COPM = Canadian Occupational Performance Meausure; CPQOL-Child = Cerebral Palsy Quality of Life Questionnaire for Children; ENNAS = Einstein Neonatal Neurobehavioral Assessment Scale; ETCH = Evaluation Tool of Children's Handwriting; FES Family Empowerment Scale; fTORT = functional Tactile Object Recognition Test; GAS = Goal Attainment Scale; GMA = General Movements Assessment; GMFCS $=$ Gross Motor Function Classification System; GMFM = Gross Motor Function Measure; GMFM- 66 = Gross Motor Function Measure - 66; GMFM-88 = Gross Motor Function Measure - 88; ICFI = International Classification of Functioning Interview; JTTHF = Jebsen-Taylor Hand Function Test; LIFE-H = Assessment of Life Habits; MA2 = Melbourne Assessment 2; MACS = Manual Ability Classification System; MAS = Modified Ashworth Scale; Melbourne = Melbourne Assessment of Unilateral Upper Limb Function; MHA = Minnesota Handwriting Assessment; NHDC = Neurological Hand Deformity Classification; NSMDA = Neurosensory Motor Developmental Assessment; PAC $=$ Preferences for Activity of Children; PAFT = Pediatric Arm Function Test; PDMS-2 = Peabody Developmental Motor Scales II; PEDI = Pediatric Evaluation of Disability Inventory; PedsQL = Pediatric Quality of Life Inventory; PGMS = Peabody Gross Motor Scale; PMAL = Pediatric Motor Activity Log; PQRS = Performance Quality Rating Scale; PRT = Pediatric Reach Test; PSI-Short form = Parenting Stress Index-Short Form; QUEST = Quality of Upper Extremity Skills Test; ROM = Range of Motion; SAS = Sitting Assessment Scale; SIPT $=$ Sensory Integration and Praxis Test; SPPC $=$ Harter Self-Perception Profile; SSC $=$ Sense and Self-Regulation Checklist; SWMs = Semmes Weinstein Monofilaments; TAUT = Toddler Arm Use Test; TCMS = Trunk Control Measurement Scale; THS-R = Test of Handwriting Skills-Revised; TIMP = Test of Infant Motor Performance; TIS = Trunk Impairment Scale; TVPS3 = Test of Visual Perceptual Skill -non-motor; VAS = Visual Analogue Scale; VMI = Beery-Buktenica Developmental Test of Visual-Motor Integration; VOAA = Video Observations Aarts and Aarts].
\end{abstract}

Melbourne Assessment of Unilateral Upper Limb Function (MUUL), Pediatric Motor Activity Log (PMAL), Quality of Upper Extremity Skills Test (QUEST), and Jebsen-Taylor Hand Function Test (JTHFT). Table 2 lists these ten assessments and their associated properties.

Additionally, a prominent trend was observed in which a majority of the articles utilized more than one assessment. Specifically, 66 out of the 76 articles used two or more assessments. Using more than one assessment shows the complexity and need of assessing the unique skills and behaviors of children with CP.

\section{Discussion}

This integrative review depicted the current trend of assessments used in research involving OT interventions for children with CP. The evidence determined the AHA, PEDI, GMFCS, COPM, GAS, MACS, MUUL, PMAL, QUEST, and JTTHF, to be the most frequently used ten assessments in research in descending order. Additionally, 66 out of 76 articles used a combination of two or more assessments. Since CP is a complex condition affecting several functional domains, using more than one assessment allowed the researchers to address the 


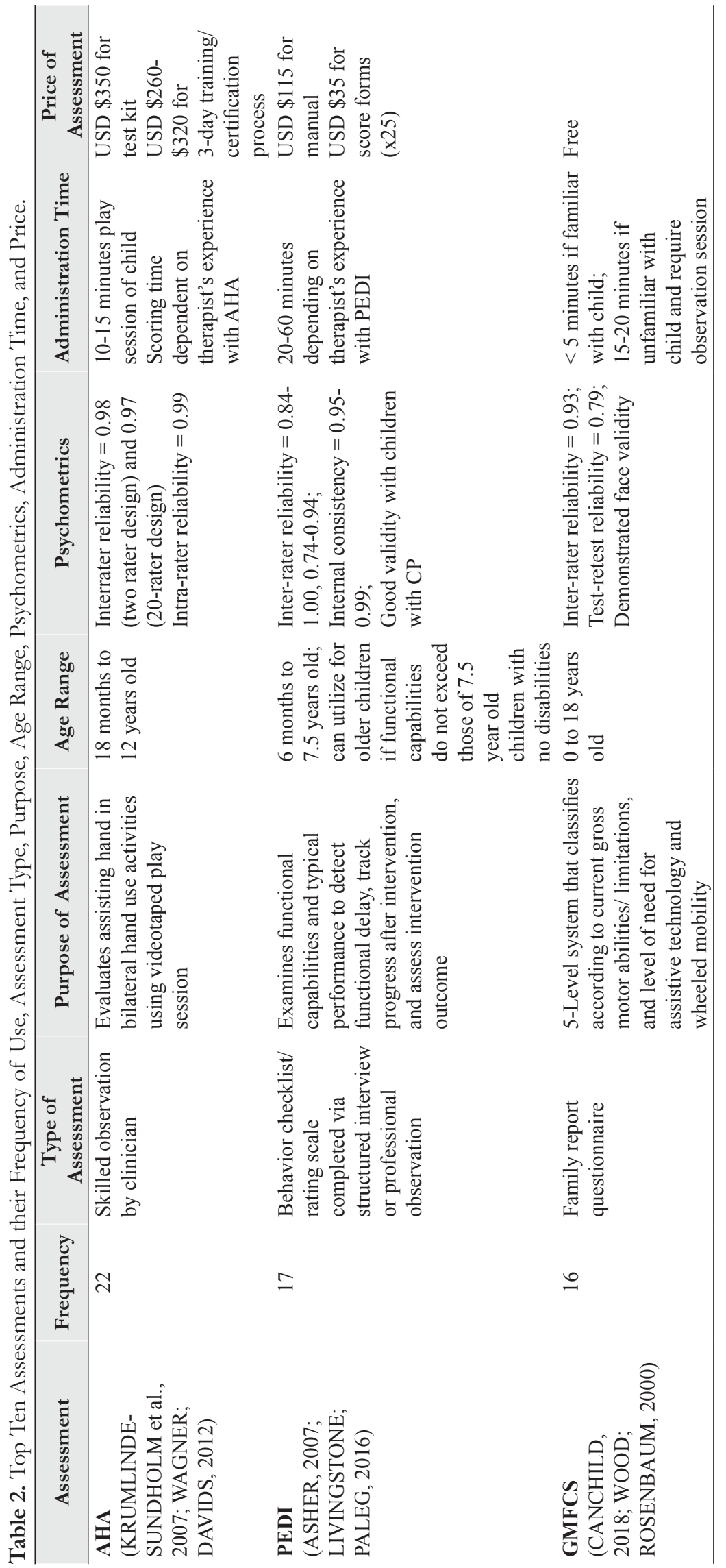




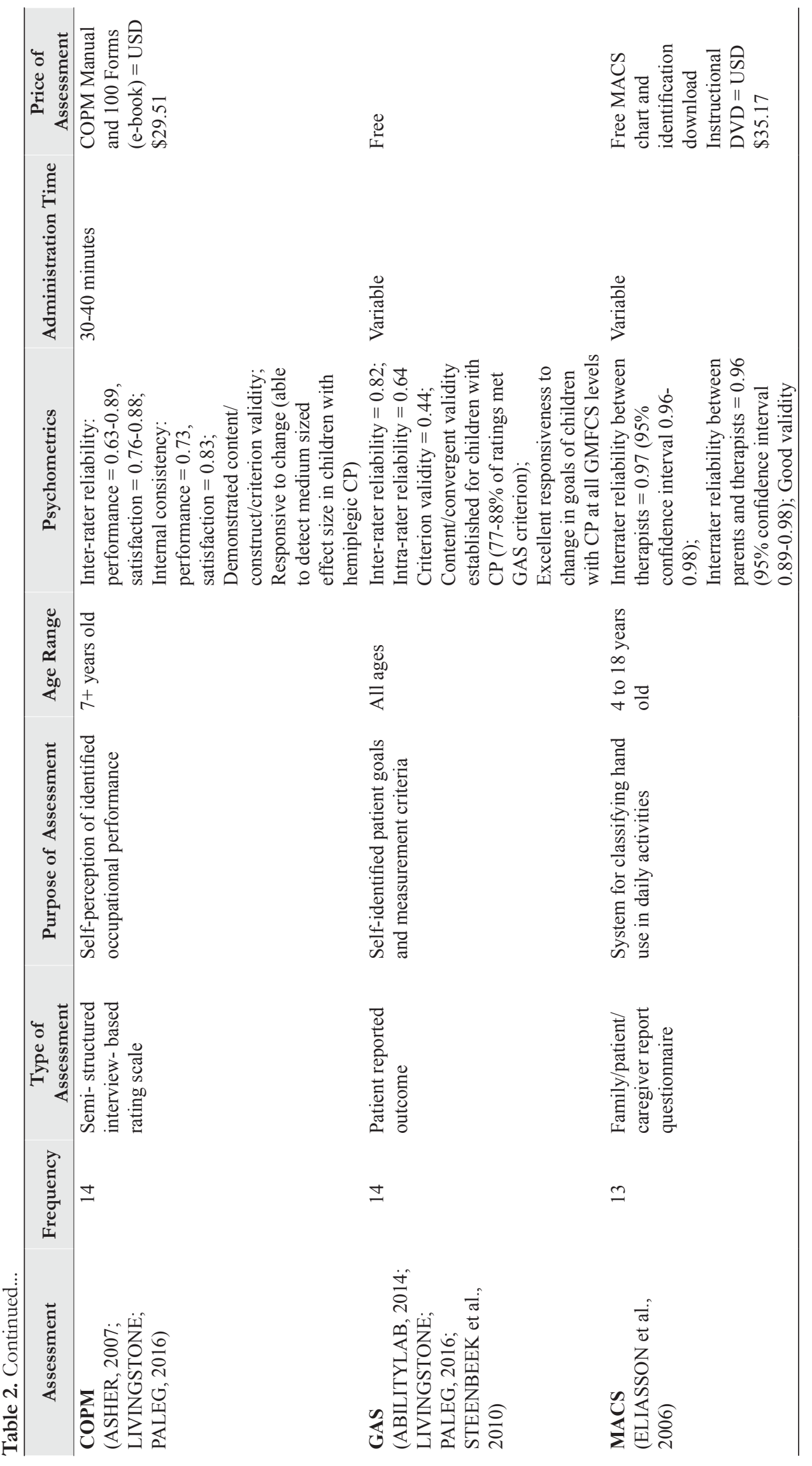




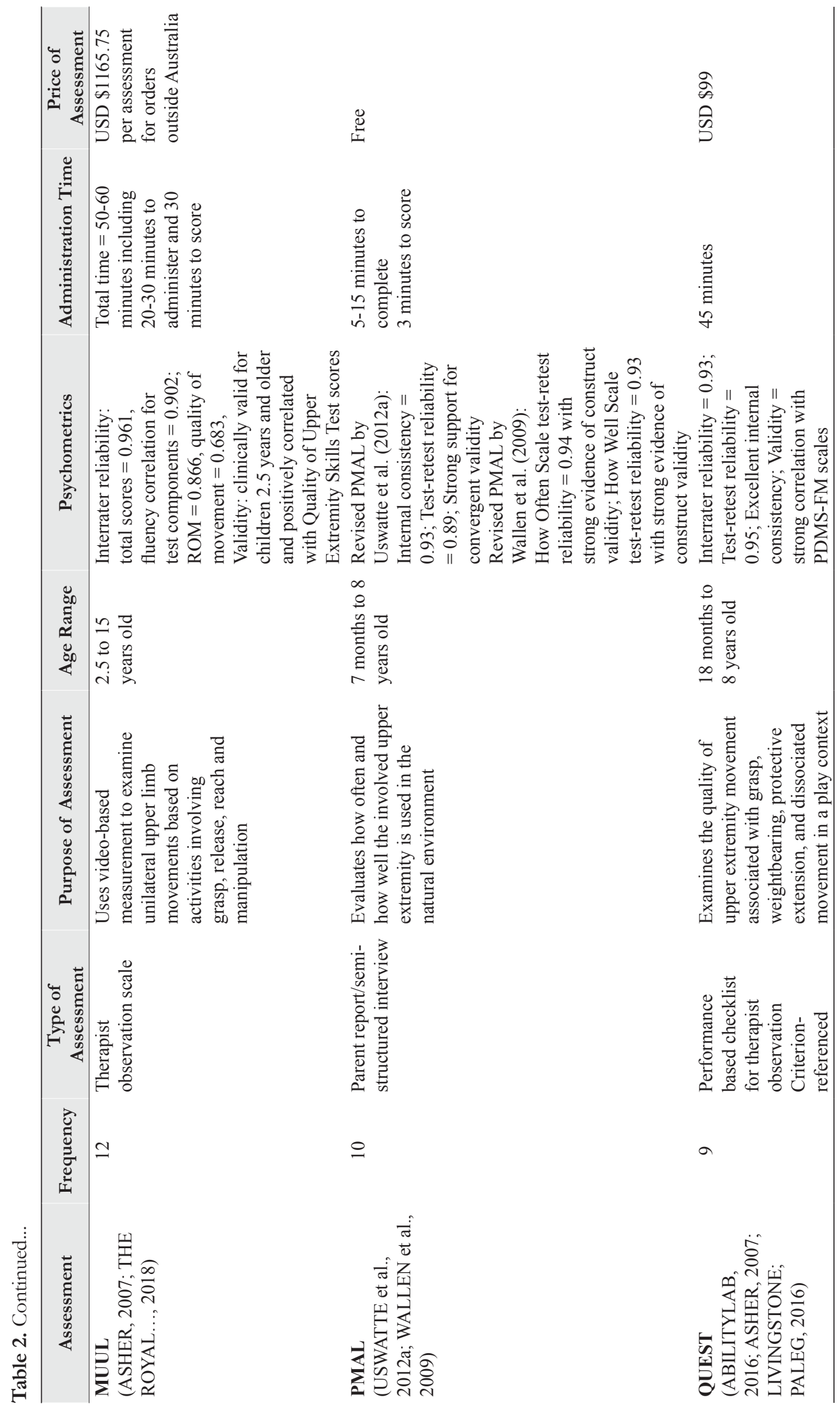




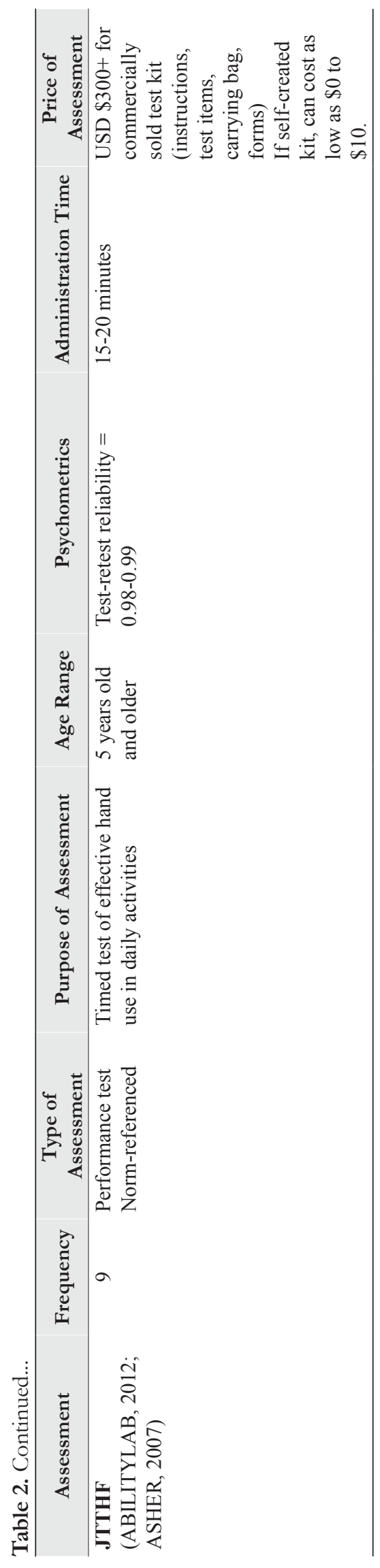


multiple needs of this population. Additionally, the use of assessments in clinical practice guides OTs not only during interventions, but also to establish therapeutic goals (BREWER; POLLOCK; WRIGHT, 2014)

The findings of this study have the following implications for OT practice:

- Because OT is a field striving towards evidence-based practice, OT practitioners working with children with $\mathrm{CP}$ can utilize the evidence from this article to aid their selection of assessments for use in clinical practice.

- With this growing focus on evidence-based practice, OT practitioners should conduct research that adds to the development of a universal toolbox to establish a standard approach of measuring OT intervention outcomes for children with CP.

Data from this study helped to establish the most frequently used assessments in research studies. Further investigation is needed to determine what assessments are currently used in clinical OT practices in order to develop a toolbox of assessments for children with CP. For instance, AHA, represents the only standardized tool of spontaneous play that measures bimanual hand use. This is a unique scope of use, which may have contributed to its frequent selection by researchers despite the high cost for the test kit and certification process (HOLMEFUR; KRUMLINDE-SUNDHOLM; ELIASSON, 2007; KRUMLINDE-SUNDHOLM et al., 2007). The second most frequented cited, the PEDI, allows a wide scope of use by researchers since it can be utilized as an initial, interim, discharge, and evaluation assessment for individual or group interventions, and rehabilitative and therapeutic programs (ASHER, 2007).

Possible factors that may have contributed to the selection of assessments in these research studies include attributes such as scope of use, affordability, feasibility with time, and psychometric properties. All of the top 10 assessments present fair to excellent psychometric properties, they are usually performed on average of 30-40 minutes, and five out of ten assessments (GMFM, COPM, GAS, PMAL, and JTTHF) cost $\$ 0$ to $\$ 40$. Measures that are accepted into a toolbox are expected to have demonstrated strong reliability and validity when use with children with cerebral palsy (STRATFORD; RIDDLE, 2005). As in these research studies, it is important for clinical OTs to also take into consideration the use of quality assessment measures to implement evidence-based practice. Therefore the use of a toolbox containing OT assessments specific for children with $\mathrm{CP}$ will ultimately enable a universal approach to guide clinicians in the rehabilitative community to measure outcomes and gauge effectiveness of their treatment.

\section{Conclusion}

These research studies showed the complexity of assessing children with CP. The clinical OTs must be aware of these complexities and that more than one assessment may be needed to capture the unique skills and behaviors of the children with CP.

As for study limitations, only two databases (PubMed and OVID) were used to search for articles that contain the targeted keywords. Utilizing more databases can widen the scope of search for studies that contain assessments and strengthen the evidence by increasing the frequency count. Additionally, even though a good portion of the articles originated from countries other than the United States, the reviewing of English-language only articles excluded data that could have provided a more accurate depiction of assessments used for children with $\mathrm{CP}$ on a global scale. Future research studies are needed to expand on the current study, to provide additional justifications for assessment use, and contribute to the development of an assessment toolbox for children with CP that is specific to measure outcomes for OT interventions.

\section{References}

AARTS, P. B. et al. A pilot study of the video observations aarts and aarts (VOAA): A new software program to measure motor behaviour in children with cerebral palsy. Occupational Therapy International, London, v. 14, n. 2, p. 113-122, 2007.

AARTS, P. B. et al. The Pirate Group Intervention Protocol: Description and a Case Report of a Modified Constraint induced Movement Therapy Combined with Bimanual Training for Young Children with Unilateral Spastic Cerebral Palsy. Occupational Therapy International, London, v. 19, n. 2, p.76-87, 2012.

ABILITYLAB. Goal Attainment Scale. Chicago, 2014. Available from: <https://www.sralab.org/rehabilitationmeasures/goal-attainment-scale\#cerebral-palsy>. Access in: 15 nov. 2018.

ABILITYLAB. Jebsen Hand Function Test. Chicago, 2012. Available from: <https://www.sralab.org/rehabilitationmeasures/jebsen-hand-function-test $>$. Access in: 15 nov. 2018. 
ABILITYLAB. Quality of Upper Extremity Skills Tests. Chicago, 2016. Available from: <https://www.sralab.org/ rehabilitation-measures/quality-upper-extremity-skills-test>. Access in: 15 nov. 2018.

\section{AMERICAN OCCUPATIONAL THERAPY} ASSOCIATION - AOTA. AOTA's Centennial Vision and executive summary. American Journal of Occupational Therapy, Bethesda, v. 61, p. 613-614, 2017. http://dx.doi. org/10.5014/ajot.61.6.613.

ARKSEY, H.; O'MALLEY, L. Scoping studies: towards amethodological framework. International Journal of Social Research Methodology, London, v. 8, n. 1, p. 19-32, 2005.

ASHER, I. E. Asher's occupational therapy assessment tools: An annotated index. Bethesda: American Occupational Therapy Association, 2007.

AULD, M. L. et al. Reproducibility of tactile assessments for children with unilateral cerebral palsy. Physical \& Occupational Therapy in Pediatrics, London, v. 32, n. 2, p. 151-166, 2012.

BAILES, A. F. et al. The effect of suit wear during an Intensive Therapy Program in children with cerebral palsy. Pediatric Physical Therapy, Batimore, v. 23, n. 2, p. 136-142, 2011.

BAILES, A. F.; GREVE, K.; SCHMITT, L. C. Changes in two children with cerebral palsy after intensive suit therapy: A case report. Pediatric Physical Therapy, Baltimore, v. 22, n. 1, p. 76-85, 2010.

BARROSO, P. N. et al. Improvement of hand function in children with cerebral palsy via an orthosis that provides wrist extension and thumb abduction. Clinical Biomechanics, Bristol, v. 26, n. 9, p. 937-943, 2011.

BERGE, S.R.T. et al. A systematic evaluation of the effect of thumb opponens splints on hand function in children with unilateral spastic cerebral palsy. Clinical Rehabilitation, London, v. 26, n. 4, p. 362-371, 2012.

BLEYENHEUFT, Y. et al. Hand and arm bimanual intensive therapy including lower extremity (HABITILE) in children with unilateral spastic cerebral palsy: A randomized trial. Neurorehabilitation and Neural Repair, New York, v. 29, n. 7, p. 645-657, 2015.

BLEYENHEUFT, Y. et al. Measuring changes of manual ability with ABILHAND Kids following intensive training for children with unilateral cerebral palsy. Developmental Medicine and Child Neurology, London, v. 59, n. 5, p. 505-511, 2017.

BRANDÃO, M. B. et al. Adapted version of constraintinduced movement therapy promotes functioning in children with cerebral palsy: A randomized controlled trial. Clinical Rehabilitation, London, v. 24, n. 7, p. 639-647, 2010.

BRANDĀO, M. B.; GORDON, A. M.; MANCINI, M. C. Functional impact of constraint therapy and bimanual training in children with cerebral palsy: A randomized controlled trial. The American Journal of Occupational Therapy, Bethesda, v. 66, n. 6, p. 672-681, 2012.
BREWER, K.; POLLOCK, N.; WRIGHT, V. Addressing the challenges of collaborative goal setting with children and their families. Physical and Occupational Therapy in Pediatrics, London, v. 34, p.138-152, 2014.

BUCCINO, G. et al. Improving upper limb motor functions through action observation treatment: A pilot study in children with cerebral palsy. Developmental Medicine \& Child Neurology, London, v. 54, n. 9, p. 822-828, 2012.

CAMERON, D. et al. Cognitive orientation to daily occupational performance (CO-OP): A new approach for children with cerebral palsy. Physical \& Occupational Therapy in Pediatrics, New York, v. 37, n. 2, p. 183-198, 2017.

CANCHILD. Gross Motor Function Classification System - Expanded \& Revised (GMFCS-E\&R). Hamilton, 2018. Available from: $<$ https://canchild.ca/en/resources/42-grossmotor-function-classification-system-expanded-revisedgmfcs-e-r>. Access in: 15 nov. 2018.

CASE-SMITH, J. et al. Multicenter randomized controlled trial of pediatric constraint-induced movement therapy: 6-month follow-up. American Journal of Occupational Therapy, Bethesda, v. 66, n. 1, p. 15-23, 2012.

CHEN, C. et al. Potential predictors of functional outcomes after home-based constraint-induced therapy for children with cerebral palsy. American Journal of Occupational Therapy, Bethesda, v. 68, n. 2, p. 159-166, 2014.

CHRISTENSEN, D. et al. Prevalence of cerebral palsy, co-occurring autism spectrum disorders, and motor functioning - Autism and Developmental Disabilities Monitoring Network, USA, 2008. Developmental Medicine and Child Neurology, London, v. 56, n. 1, p. 59-65, 2014.

COHEN-HOLZER, M. et al. The influence of a constraint and bimanual training program using a variety of modalities, on upper extremity functions and gait parameters among children with hemiparetic cerebral palsy: a case series. Physical \& Occupational Therapy in Pediatrics, London, v. 36, n. 1, p. 17-27, 2016.

COKER-BOLT, P. C.; GARCIA, T.; NABER, E. Neuromotor: Cerebral palsy. In: CASE-SMITH, J.; O'BRIEN, J. (Ed.). Occupational therapy for children and adolescents. St. Louis: Elsevier, 2015. p. 793-811.

DELUCA, S. C. et al. Constraint-induced movement therapy (CIMT) for young children with cerebral palsy: Effects of therapeutic dosage. Journal of Pediatric Rehabilitation Medicine, Amsterdam, v. 5, n. 2, p. 133-142, 2012.

DIAS, T. S. et al. As contribuiçóes da gameterapia no desempenho motor de indivíduo com paralisia cerebral. Cadernos Brasileiros de Terapia Ocupacional, São Carlos, v. 25, n. 3, p. 575-584, 2017.

ELIASSON, A. C. et al. The Manual Ability Classification System (MACS) for children with cerebral palsy: scale development and evidence of validity and reliability. Developmental Medicine \& Child Neurology, London, v. 48, n. 7, p. 549-554, 2006 
FERRE, C. L. et al. Caregiver-directed home-based intensive bimanual training in young children with unilateral spastic cerebral palsy: A randomized trial. Developmental Medicine and Child Neurology, London, v. 59, n. 5, p. 497-504, 2017.

GELKOP, N. et al. Efficacy of constraint-induced movement therapy and bimanual training in children with hemiplegic cerebral palsy in an educational setting. Physical \& Occupational Therapy in Pediatrics, New York, v. 35, n. 1, p. 24-39, 2015.

GEORGIADES, M. et al. The Neurological Hand Deformity Classification for children with cerebral palsy. Australian Occupational Therapy, Cambridgeshire, v. 61, n. 6, p. 394-402, 2014.

GOLOMB, M. R. et al. In-home virtual reality videogame telerehabilitation in adolescents with hemiplegic cerebral palsy. Archives of Physical Medicine and Rehabilitation, Philadelphia, v. 91, n. 1, p. 1-8, 2010.

GORDON, A. M.; SCHNEIDER, J. A.; CHINNAN, A. P. T. Efficacy of a hand-arm bimanual intensive therapy (HABIT) in children with hemiplegic cerebral palsy: A randomized control trial. Developmental Medicine \& Child Neurology, London, v. 49, n. 11, p. 830-838, 2007.

HAMIL, D.; WASHINGTON, K.; WHITE, O. R. The effect of hippotherapy on postural control in sitting for children with cerebral palsy. Physical \& Occupational Therapy in Pediatrics, New York, v. 27, n. 4, p. 23-42, 2007.

HANSEN, A. B. et al. Myofascial structural integration: A promising complementary therapy for young children with spastic cerebral palsy. Journal of Evidence-Based Complementary \& Alternative Medicine, Thousand Oaks, v. 17, n. 2, p. 131-135, 2012.

HARVEY, A. et al. A systematic review of measures of activity limitation for children with cerebral palsy. Developmental Medicine \& Child Neurology, London, v. 50, n. 3, p. 190-198, 2008.

HOARE, B. J. et al. Intensive therapy following upper limb botulinum toxin A injection in young children with unilateral cerebral palsy: a randomized trial. Developmental Medicine and Child Neurology, London, v. 55, n. 3, p. 238-247, 2013.

HOARE, B. J. et al. Modified constraint-induced movement therapy or bimanual occupational therapy following injection of botulinum toxin-A to improve bimanual performance in young children with hemiplegic cerebral palsy: A randomised controlled trial methods paper. $B M C$ Neurology, London, v. 10, n. 58, p. 1-20, 2010.

HOLMEFUR, M.; KRUMLINDE-SUNDHOLM, L.; ELIASSON, A. C. Interrater and intrarater reliability of the assisting hand assessment. American Journal of Occupational Therapy, Bethesda, v. 61, n. 1, p. 79-84, 2007.

HOUWINK, A. et al. Assessment of upper limb capacity, performance, and developmental disregard in children with cerebral palsy: validity and reliability of the revised Video-Observation Aarts and Aarts module: Determine Developmental Disregard (VOAA-DDD-R). Developmental
Medicine and Child Neurology, London, v. 55, n. 1, p. 76-82, 2013

HOWCROFT, J. et al. Wearable wrist activity monitor as an indicator of functional hand use in children with cerebral palsy. Developmental Medicine \& Child Neurology, London, v. 53, n. 11, p. 1024-1029, 2011.

HUANG, H. et al. Modified toy cars for mobility and socialization: case report of a child with cerebral palsy. Pediatric Physical Therapy, London, v. 26, n. 1, p. 76-84, 2014.

IMMS, C. et al. Leisure participation-preference congruence of children with cerebral palsy: a Children's Assessment of Participation and Enjoyment International Network descriptive study. Developmental Medicine of Child Neurology, London, v. 59, n. 4, p. 380-387, 2017.

JAMES, S. et al. Randomized controlled trial of web-based multimodal therapy for unilateral cerebral palsy to improve occupational performance. Developmental Medicine \& Child Neurology, London, v. 57, n. 6, p. 530-538, 2015a.

JAMES, S. et al. Test-retest reproducibility of the assessment of motor and process skills in children with unilateral cerebral palsy. Physical \& Occupational Therapy in Pediatrics, New York, v. 36, n. 2, p. 144-154, 2015 b.

KARA, O. K. et al. The effects of Kinesio Taping on body functions and activity in unilateral spastic cerebral palsy: a single-blind randomized controlled trial. Developmental Medicine \& Child Neurology, London, v. 57, n. 1, p. 81-88, 2015.

KETELAAR, M.; VERMEER, A.; HELDERS, P. J. Functional motor abilities of children with cerebral palsy: a systematic literature review of assessment measures. Clinical Rehabilitation, London, v. 12, n. 5, p. 369-380, 1998.

KIRKPATRICK, E. et al. Effect of parent-delivered action observation therapy on upper limb function in unilateral cerebral palsy: A randomized controlled trial. Developmental Medicine \& Child Neurology, London, v. 58, n. 10, p. 1049-1056, 2016.

KRUIJSEN-TERPSTRA, A. J. A. et al. Efficacy of three therapy approaches in preschool children with cerebral palsy: a randomized controlled trial. Developmental Medicine of Child Neurology, London, v. 58, n. 7, p. 758-766, 2016.

KRUMLINDE-SUNDHOLM, L. et al. The assisting hand assessment: Current evidence of validity, reliability, and responsiveness to change. Developmental Medicine of Child Neurology, London, v. 49, n. 4, p. 259-264, 2007.

LAW, M. C. et al. Focus on function: A cluster, randomized controlled trial comparing child- versus context-focused intervention for young children with cerebral palsy. Developmental Medicine \& Child Neurology, London, v. 53, n. 7, p. 621-629, 2011.

LIDMAN, G. et al. Botulinum toxin A injections and occupational therapy in children with unilateral spastic cerebral palsy: A randomized controlled trial. Developmental 
Medicine and Child Neurology, London, v. 57, n. 8, p. 754-761, 2015.

LIN, K. et al. Effects of home-based constraint-induced therapy versus dose-matched control intervention on functional outcomes and caregiver well-being in children with cerebral palsy. Research in Developmental Disabilities, New York, v. 32, n. 5, p. 1483-1491, 2011.

LIVINGSTONE, R.; PALEG, G. Measuring outcomes for children with cerebral palsy who use gait trainers. Technologies, Switzerland, v. 4, n. 3, p.1-19, 2016.

LOUWERS, A. et al. Immediate effect of a wrist and thumb brace on bimanual activities in children with hemiplegic cerebral palsy. Developmental Medicine \& Child Neurology, London, v. 53, n. 4, p. 321-326, 2011.

LOWES, L. P. et al. Pilot study of the efficacy of constraintinduced movement therapy for infants and toddlers with cerebral palsy. Physical \& Occupational Therapy in Pediatrics, London, v. 34, n. 1, p. 4-21, 2014.

LUNA-OLIVA, L. et al. Kinect xbox 360 as a therapeutic modality for children with cerebral palsy in a school environment: A preliminary study. NeuroRehabilitation, Amsterdam, v. 33, n. 4, p. 513-521, 2013.

MACKEY, A. H. et al. Use of three-dimensional kinematic analysis following upper limb botulinum toxin A for children with hemiplegia. European Journal of Neurology, London, v. 15, n. 11, p. 1191-1198, 2008.

MAN, D. W. K.; WONG, M. L. Evaluation of computeraccess solutions for students with quadriplegic athetoid cerebral palsy. American Journal of Occupational Therapy, Bethesda, v. 61, n. 3, p. 355-364, 2007.

MATUSIAK-WIECZOREK, E.; MAŁACHOWSKASOBIESKA, M.; SYNDER, M. Influence of hippotherapy on body balance in the sitting position among children with cerebral palsy. Ortopedia, Traumatologia, Rehabilitacja, Warszawa, v. 18, n. 2, p. 165-175, 2016.

MCCONNELL, K.; JOHNSTON, L.; KERR, C. Efficacy and acceptability of reduced intensity constraintinduced movement therapy for children aged 9-11 years with hemiplegic cerebral palsy: A pilot study. Physical \& Occupational Therapy in Pediatrics, New York, v. 34, n. 3, p. 245-259, 2014.

MCGARRY, S.; MOIR, L.; GIRDLER, S. The Smart Wheelchair: is it an appropriate mobility training tool for children with physical disabilities? Disabil Rehabil Assist Technol, England, v. 7, n. 5, p. 372-380, 2012.

MCLEAN, B. et al. Somatosensory discrimination intervention improves body position sense and motor performance in children with hemiplegic cerebral palsy. American Journal of Occupational Therapy, Bethesda, v. 71, n. 3, p. 7103190060p1-7103190060p9, 2017.

MILlARD, E.; BENORE, E.; MOSHER, K. A multidisciplinary functional toileting pathway for children with cerebral palsy: Preliminary analysis. Clinical Practice in Pediatric Psychology, Washington, v. 1, n. 1, p. 81-88, 2013.

NOVAK, I.; CUSICK, A.; LOWE, K. A pilot study on the impact of occupational therapy home programming for young children with cerebral palsy. American Journal of Occupational Therapy, Bethesda, v. 61, n. 4, p. 463468, 2007.

OSTENSJØ, S.; OIEN, I.; FALLANG, B. Goal-oriented rehabilitation of preschoolers with cerebral palsy--a multicase study of combined use of the canadian occupational performance measure (COPM) and the goal attainment scaling (GAS). Developmental Neurorehabilitation, London, v. 11, n. 4, p. 252-259, 2008.

PALSBO, S. E.; HOOD-SZIVEK, P. Effect of roboticassisted three-dimensional repetitive motion to improve hand motor function and control in children with handwriting deficits: A nonrandomized phase 2 device trial. The American Journal of Occupational Therapy, Bethesda, v. 66, n. 6, p. 682-690, 2012.

PHAM, H. P. et al. Validity and responsiveness of the trunk impairment scale and trunk control measurement scale in young individuals with cerebral palsy. Physical o Occupational Therapy in Pediatrics, New York, v. 36, n. 4, p. 440-452, 2016.

PHIPPS, S.; ROBERTS, P. Predicting the effects of cerebral palsy severity on self-care, mobility, and social function. American Journal of Occupational Therapy, Bethesda, v. 66, n. 4, p. 422-429, 2012

PIERNIK-YODER, B.; BECK, A. The use of standardized assessments in occupational therapy in the United States. Occupational Therapy in Health Care, London, v. 26, n. 2-3, p. 97-108, 2012.

PSYCHOULI, P.; KENNEDY, C. R. Modified constraintinduced movement therapy as a home-based intervention for children with cerebral palsy. Pediatric Physical Therapy, London, v. 28, n. 2, p.154-160, 2016.

ROBERT, M. T. et al. Motor learning in children with hemiplegic cerebral palsy and the role of sensation in short-term motor training of goal-directed reaching. Developmental Medicine \& Child Neurology, London, v. 55, n. 12, p. 1121-1128, 2013.

ROSTAMI, H. R. et al. Effects of modified constraint-induced movement therapy in virtual environment on upper-limb function in children with spastic hemiparetic cerebral palsy: A randomised controlled trial. NeuroRehabilitation, Amsterdam, v. 31, n. 4, p. 357-365, 2012.

RYAN, S. E.; RIGBY, P. J.; CAMPBELL, K. A. Randomised controlled trial comparing two school furniture configurations in the printing performance of young children with cerebral palsy. Australian Occupational Therapy Journal, Cambridgeshire, v. 57, n. 4, p. 239-245, 2010.

RYLL, U. C.; BASTIAENEN, C. H.; ELIASSON, A. Assisting hand assessment and children's hand-use experience questionnaire -observed versus perceived bimanual performance in children with unilateral cerebral palsy. Physical \& Occupational Therapy in Pediatrics, New York, v. 37, n. 2, p. 199-209, 2017.

SALEH, M. N. et al. Actual vs. best practices for young children with cerebral palsy: A survey of paediatric occupational therapists and physical therapists in Quebec, 
Canada. Developmental Neurorehabilitation, London, v. 11, n. 1, p. 60-80, 2009.

SAKZEWSKI, L. et al. Equivalent Retention of Gains at 1 Year After Training With Constraint-Induced or Bimanual Therapy in Children With Unilateral Cerebral Palsy. Neurorehabilitation and Neural Repair, New York, v. 25, n. 7, p. 664-671, 2011.

SAKZEWSKI, L. et al. Impact of intensive upper limb rehabilitation on quality of life: A randomized trial in children with unilateral cerebral palsy. Developmental Medicine \& Child Neurology, London, v. 54, n. 5, p. 415-423, 2012.

SAKZEWSKI, L. et al. Randomized comparison trial of density and context of upper limb intensive group versus individualized occupational therapy for children with unilateral cerebral palsy. Developmental Medicine of Child Neurology, London, v. 57, n. 6, p. 539-547, 2015.

SCHNEIBERG, S. et al. The effectiveness of task-oriented intervention and trunk restraint on upper limb movement quality in children with cerebral palsy. Developmental Medicine \& Child Neurology, London, v. 52, n. 11, p. e245-e253, 2010

SCHRANK, J. P. T. DPT. Constraint-induced movement therapy effects on gross motor function of a child with triplegic cerebral palsy. Pediatric Physical Therapy, Baltimore, v. 25, n. 1, p. 71-78, 2013.

SILVA, L. M. T. et al. Qigong massage for motor skills in young children with cerebral palsy and down syndrome. American Journal of Occupational Therapy, Bethesda, v. 66, n. 3, p. 348-355, 2012.

SNIDER, L. M. et al. A comparison of the general movements assessment with traditional approaches to newborn and infant assessment: Concurrent validity. Early Human Development, Limerick, v. 84, n. 5, p. 297-30, 2008.

STEENBEEK, D. et al. Interrater reliability of goal attainment scaling in rehabilitation of children with cerebral palsy. Archives of Physical Medicine and Rehabilitation, Philadelphia, v. 91, n. 3, p. 429-435, 2010.

STEULTJENS, E. M. et al. Occupational therapy for children with cerebral palsy: A systematic review. Clinical Rehabilitation, London, v. 18, n. 1, p.1-14, 2004.

STORVOLD, G. V. P. T.; JAHNSEN, R. P. T. Intensive motor skills training program combining group and individual sessions for children with cerebral palsy. Pediatric Physical Therapy, Baltimore, v. 22, n. 2, p. 150-159, 2010.

STRATFORD, P.; RIDDLE, D. Assessing sensitivity to change: choosing the appropriate change coefficient. Health Qual Life Outcome, London, v. 3, n. 23, p. 1-7, 2005.

THE ROYAL CHILDREN'S HOSPITAL MELBOURNE. How to order the Melbourne Assessment 2. Australia, 2018. Available from: <https://www.rch.org.au/melbourneassessment/ how-to-order/>. Access on: 15 nov. 2018.

THOMPSON, A. M. et al. Constraint-induced movement therapy in children aged 5 to 9 years with cerebral palsy: A day camp model. Pediatric Physical Therapy, Baltimore, v. 27, n. 1 , p. $72-80,2015$.
USWATTE, G. et al. Pediatric arm function test: Reliability and validity for assessing more-affected arm motor capacity in children with cerebral palsy. American Journal of Physical Medicine \& Rehabilitation, Baltimore, v. 91, n. 12, p. 1060-1069, 2012a.

USWATTE, G. et al. The Pediatric Motor Activity LogRevised: Assessing real-world arm use in children with cerebral palsy. Rehabilitation Psychology, Washington, v. 57, n. 2, p. 149-158, 2012 b.

WAGNER, L. V.; DAVIDS, J. R. Assessment tools and classification systems used for the upper extremity in children with cerebral palsy. Clinical Orthopaedics and Related Research, Philadelphia, v. 470, n. 5, p. 1257-1271, 2012.

WALLEN, M. et al. Modified constraint-induced therapy for children with hemiplegic cerebral palsy: A feasibility study. Developmental Neurorehabilitation, London, v. 11, n. 2, p. 124-133, 2008.

WALLEN, M. et al. Modified constraint-induced therapy for children with hemiplegic cerebral palsy: A randomized trial. Developmental Medicine and Child Neurology, Philadelphia, v. 53, n. 12, p. 1091-1099, 2011.

WALLEN, M. et al. Psychometric properties of the Pediatric Motor Activity Log used for children with cerebral palsy. Developmental Medicine \& Children Neurology, London, v. 51, n. 3, p. 200-208, 2009.

WALLEN, M.; O'FLAHERTY, S. J.; WAUGH, M. A. Functional outcomes of intramuscular botulinum toxin type a and occupational therapy in the upper limbs of children with cerebral palsy: A randomized controlled trial. Archives of Physical Medicine and Rehabilitation, Philadelphia, v. 88, n. 1, p. 1-10, 2007.

WANG, T. et al. Psychometric and clinimetric properties of the melbourne assessment 2 in children with cerebral palsy. Archives of Physical Medicine \& Rehabilitation, Philadelphia, v. 98, n. 9, p. 1836-1841, 2017.

WRIGHT, F. V.; MAJNEMER, A. The concept of a toolbox of outcome measures for children with cerebral palsy: Why, what, and how to use? Journal of Child Neurology, Littleton, v. 29, n. 8, p. 1055-1065, 2014.

YABUNAKA, Y. et al. Evaluating the effect of intensive intervention in children with cerebral palsy using a hypothetical matched control group: A preliminary study. American Journal of Physical Medicine \& Rehabilitation, Baltimore, v. 90, n. 2, p. 128-136, 2011.

YASUKAWA, A.; URONIS, J. Effectiveness of the dynamic movement orthosis glove for a child with cerebral palsy hemiplegia and obstetric brachial plexus palsy: A case series. JPO Journal of Prosthetics \& Orthotics, United States, v. 26, n. 2, p. 107-112, 2014

ZIEBELL, M. et al. The relationship between physical performance and self-perception in children with and without cerebral palsy. Australian Occupational Therapy Journal, Cambridgeshire, v. 56, n. 1, p. 24-32, 2009. 


\section{Author's Contributions}

Carly Peters - took the leadership role in searching for the articles, organizing the result and discussion sections. Amy Chang - Assited with the articles search, result and discussion sections. Abigail Morales - Assited with the articles search, result and discussion sections. Karin Barnes - had a consultant role due to her expertise in pediatrics. Ana Allegretti - is the academic mentor of the students and principal investigator of the research study. All authors approved the final version of the text. 